ISSN: 1813-162X (Print) ; 2312-7589 (Online)
available online at: http://www.tj-es.com

\title{
Developing Recognition System for New Iraqi License Plate
}

\section{Ghassan Khazal Ali * \\ Computer and Software Department College of Engineering \\ Diyala University \\ Diyala \\ Iraq}

\section{Keywords:}

\section{Recognition}

License plate recognition (LPR)

Optical character recognition (OCR)

Label plate number (LPN)

Image processing

\section{A R T I C L E I N F O}

\section{Article history:}

Received

10 October 2016

Accepted

23 October 2017

Available online 11 March 2018

\author{
A B S T R A C T
}

Because of rapid population growth and increasing need for humans to use the vehicles in the last decade. Identifying these vehicles by license plates is considered. The quick development in image processing field can solve this issue by capturing the image of these vehicles and then identify the vehicle license plate. Most traffic applications depend on Automatic detection of the license plate and identification technology such as searching for stolen vehicles, traffic control on the road, monitoring cars from entering area, some information about the vehicle, parking systems, monitor the border crossing, maximum speed or red-light violation ticket, and identify the identity of the driver etc. In this paper we try to design a system that can identify the characters of the new plates of vehicles in Iraq, which relies on image recognition. First capture the picture of the vehicle using a digital camera and recognize the text of the captured pictures using optical character recognition technology and then compared it with all vehicle plates numbers stored in the database.

(c) 2018 TJES, College of Engineering, Tikrit University

DOI: http://dx.doi.org/10.25130/tjes.25.1.02

تصميم نظام للتعرف على لوحات تسجيل السيارات العراقية الجديدة

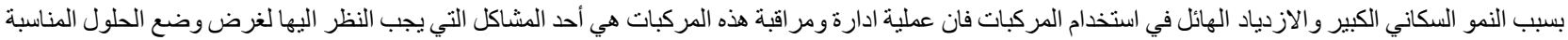

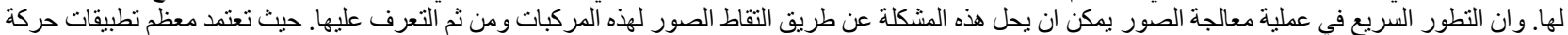

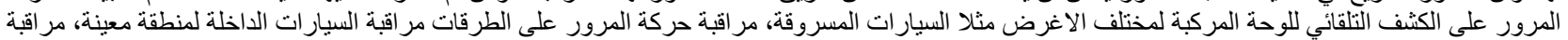

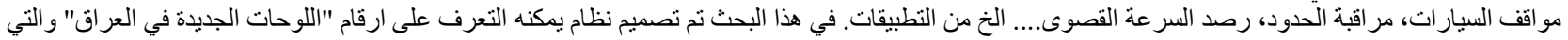

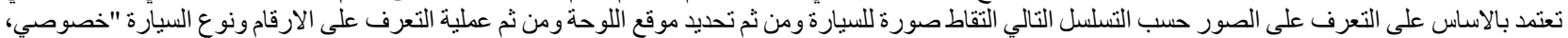
حمل ... الخ " و المحافظة التي تتبع لها السيارة.

\section{INTRODUCTION}

License Pate Recognition (LPR) is a very important application which has become one of the regulations applied in many different areas of life because of its significant role in work achievement, save time, decrease man power and provide high accuracy such as in the control systems of own garages and state institutions, in providing fuel stations or search for stolen cars and even required in. LPR was designed to read the vehicle's plate characters. Capturing a plate image and then recognizing it include detection, processing, and recognition. LPR applications has advantage of the pattern recognition to identify the characters on plates. The system consists of the following parts: a camera, image processor, and a computer that save and read the plates images. The license plate systems may be based on Artificial Neural Network [1], Probabilistic Neural Network [2], Optical Character Recognition (3), Feature salient (4), Support Vector Machine (SVM) (5), region based (6), color segmentation (7), fuzzy based algorithm (8).

In this paper, the requirements and the properties of the new plate is considered, and image matching based algorithm as identification algorithm is used because of the complex shape of the Iraq new plate. In this application we use image processing and pattern recognition techniques. The system may consist of the following steps:

1. Vehicle image capturing.

2. Plate image extracting. 
3. License plate characters extracting.

4. Characters recognizing.

This paper consists of six sections; section two reviews the related works, section three discus the application of the system, section four for experiment results, section five and six conclusion and references.

\section{RELATED WORK}

Christos et al. [1] present a new algorithm for vehicle license plate identification, basis on a novel adaptive image segmentation technique and connected component in conjunction with a character recognition. Fikriye and Figen [2], design an algorithm that can recognize plates using the pictures taken at various angles, various distances and different times of the day. And The locate the plate using Otsu's thresholding method. Roy and Ghosha [3] try to solve Automatic Number Plate Recognition (ANPR) problem by using a pixel based segmentation algorithm. Zhen et al. [4], use novel method to recognize plate characters. By uses a feature-salience classifier. Kamal [9], include three basic stages (preprocessing, license plate localization and license plate recognition). Jones et al. [10], present a face detection framework that is capable of processing images extremely rapidly while achieving high detection rates is proposed. In this work a template matching algorithm has been applied for recognition after preprocessing the captured image and get the location of the plate.

\section{SYSTEM ARCHITECTURE}

The general form of the license plate system is given in Fig. 1 which performs the following main steps:

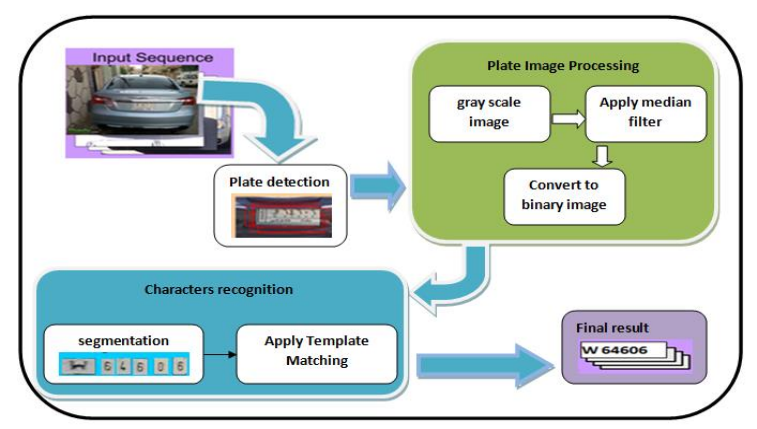

Fig. 1. Proposed system steps.

\subsection{Image Acquisition}

The process of image capturing using camera is called image acquisition which represent the first step in the system. The type of camera that used in this stage is important since there are different properties must be considered in choosing the camera such as storage capability, the range of zoom, connectivity, mega pixels, and others. In this work, the image is loaded from computer as acquisition process then this image is preprocessed using proposed algorithm. In Iraq license plate, different forms in terms of design and color according to the field of use such as a government, private, taxis, pregnancy cars and others.

\subsection{License Plate Detection}

The detector is based on the Viola and Jones Framework [10]. The Viola-Jones frame is part of the object detection sensor at competitive precise of objects in real time are proposed. however, they designed it to detect the objects, also it used in face recognition [11].

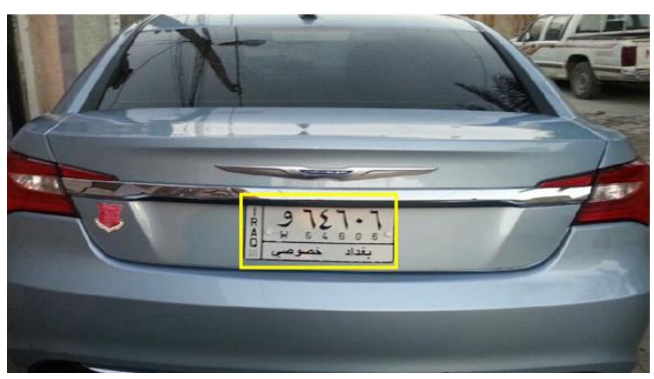

Fig. 2. License plate detection.

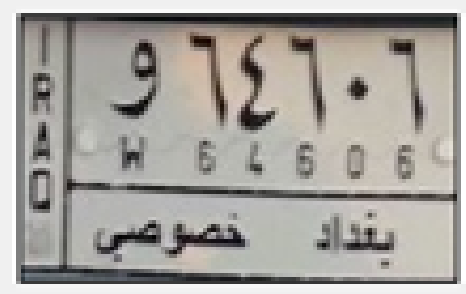

Fig. 3. Captured image.

\subsection{Plate Image Processing}

In this stage there are many operations:

\subsubsection{Grey Conversion}

In this stage convert the captured colored RGB image to a 256 grayscale by using the flowing formula (1).

$G R A Y=0.29 R+0.59 G+0.11 B$

The purpose of taking grey image in which each pixel as a number between 0-255 to remove the contrast between the colors Red, Green and Blue channels of captured image and then converting it into grey scale image.

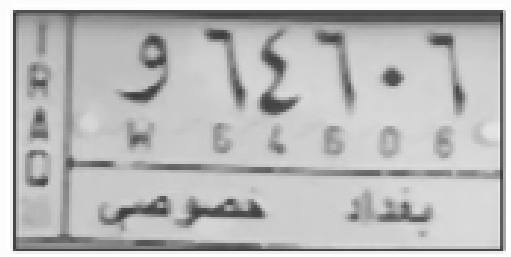

Fig. 2. Image after converted to gray scale.

\subsubsection{Median Filtering}

Median filter has been used to remove the noises. also under certain conditions, it maintains the edges during noise removal.

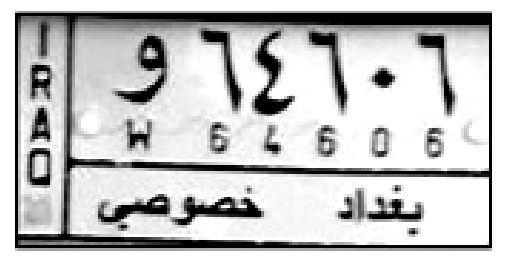

Fig. 3. Image after median filtering. 


\subsubsection{Binary Image Converting}

The Image should have converted from Gray scale into binary image. Because the Intensity value calculated easily as compared to Grayscale and color image. Binary image is shown in Fig. 4.

There we use the function from the Scilab Image Processing module to find the threshold value automatically.

th $=$ imgraythresh(LPRgray)

th $=0.6984375$

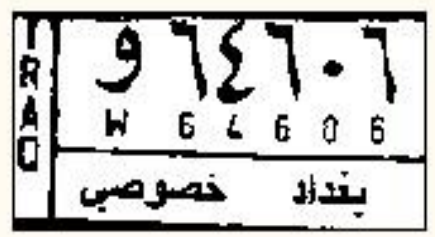

Fig. 4. Binary image.

\subsection{License Plate Recognition}

There are many steps in License plate recognition phase:

\subsubsection{Segmentation}

Segmentation mean divide the digital image into regions or sets of pixels represent different objects in the image, usually having the same color or texture [6]. In many LPR applications segmentation is an important stage. This stage may be difficult for many reasons, such as noise, plate frame, and rotation and illumination variance. The primary steps of segmentation are as shown in following flowchart:

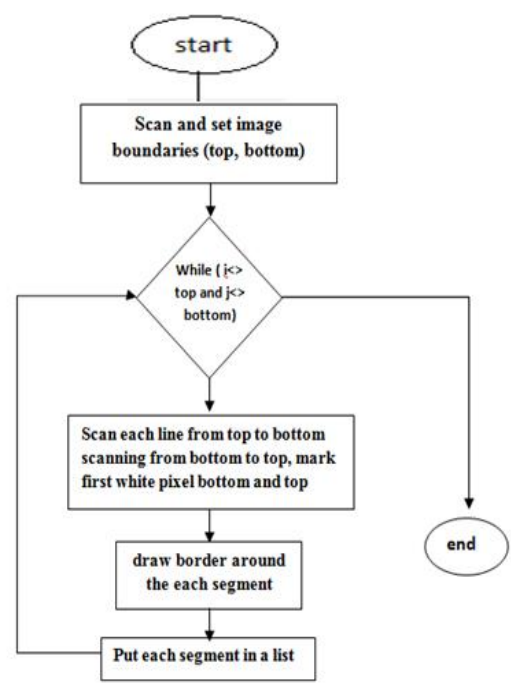

Fig. 5. Flowchart of segmentation process

The segmentation result of some license plate characters is shown in Fig. 6. modifying the samples of the audio signal directly and indirectly, such as

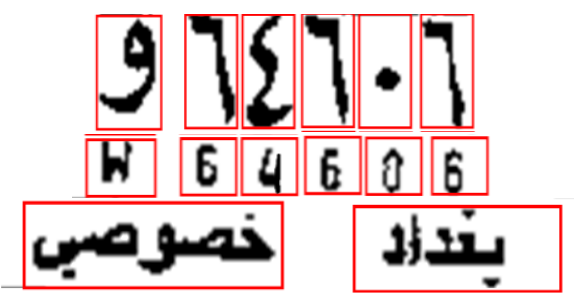

Fig. 6. License plate segmentation.

\subsubsection{License Plate Classification}

The new license plates in Iraq is consist of five parts as shown in Fig. 7:

1. The first section: represent the country name (Iraq), all car plates types contain this section.

2. The second section: $X 1 \times X 2 X 3 X 4 X 5 X 6 X 7$. Here $X 1$ is an Arabic letter $X 2-X 7$ are Indian numerals.

3. The third section: $\mathrm{Y}_{1} \times \mathrm{Y}_{2} \mathrm{Y}_{3} \mathrm{Y}_{4} \mathrm{Y}_{5} \mathrm{Y}_{6} \mathrm{Y}_{7}$. Here $Y 1$ is an English letter $Y 2-Y 7$ are Arabic numerals.

4. The fourth section: represent car type like

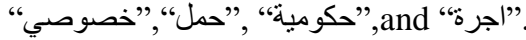

5. The fifth section: represent the province name like

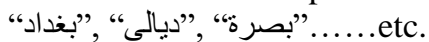

To enhance the recognition speed, we use six character classifies based on template matching. English letter classifier, Arabic letter classifier, and Arabic Numbers classifier, Indian Numbers classifier, Car type classifier, and province classifier.

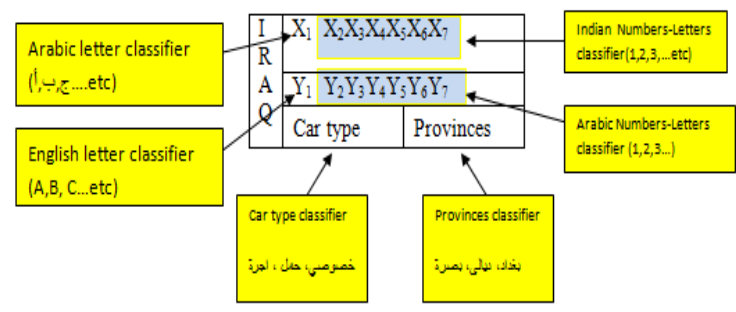

Fig. 7. Shows six types of classifiers.

\subsection{Character Recognition}

We use template based reorganization method for reorganization of individual segment. In template algorithm, each segment is compared with one image stored template image in the database. And take the best matched similarity. We design our own character image templates in our system according to the logical features of the input character segments. our template images are shown in Fig. 8, and Fig. 9 shows a flowchart of recognition process.

\begin{tabular}{|c|c|c|c|c|c|c|c|c|c|c|c|}
\hline & & j & $J$ & d & & $\dot{\tau}$ & 乙 & ? & 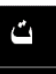 & ب & \\
\hline$\kappa$ & & $b$ & b & $\varepsilon$ & $\varepsilon$ & ف & ف & s & $J$ & ? & \\
\hline$\infty$ & g & 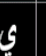 & & 1 & 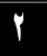 & $r$ & 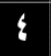 & $\theta$ & 4 & $V$ & $\Lambda$ \\
\hline 9 & ي بغال| & لديال & بصراز & ميسان & الشتح & وصل & نجل & صكوليا & فصوص & حلر & \\
\hline A & B & C & D & $E$ & $F$ & G & H & I & J & K & \\
\hline M & $\mathbf{N}$ & 0 & $\mathbf{P}$ & $Q$ & R & $S$ & $\mathrm{~T}$ & U & V & W & $X$ \\
\hline$y$ & Z & 0 & 1 & 2 & & 4 & 5 & 6 & 7 & 8 & \\
\hline
\end{tabular}

Fig. 8. Template images.

\section{EXPERIMENTAL RESULTS}

Proposed program was implemented using Microsoft visual studio 2010, we use "visual basic.net" with Matlab by using Microsoft COM "Component Object Model" interface. The vehicles Images are taken from images stored in database. The experiments applied on vehicle of deferent colors, location, and distance. we used 30 image and the program success to identify 26 plates number and 
some plates missed some numbers and the program couldn't have recognized and hence, the accuracy of the proposed method is $87 \%$ and this may be a good result as shown in Fig. 12.

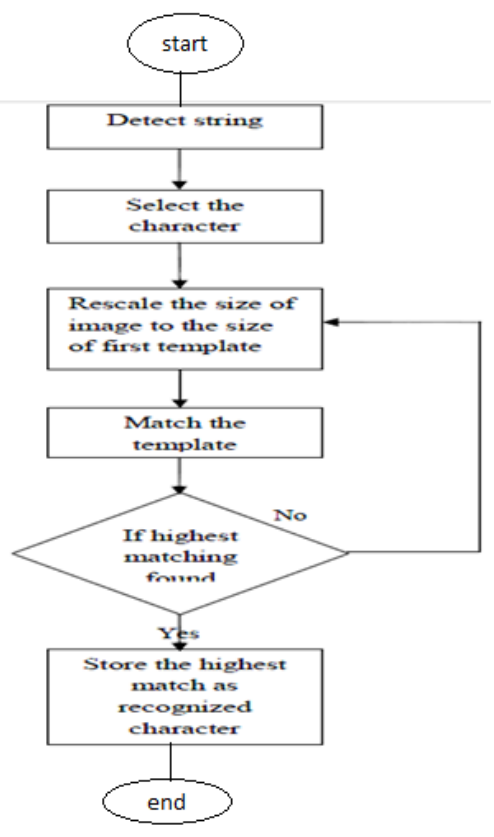

Fig. 9. Flowchart of recognition process.

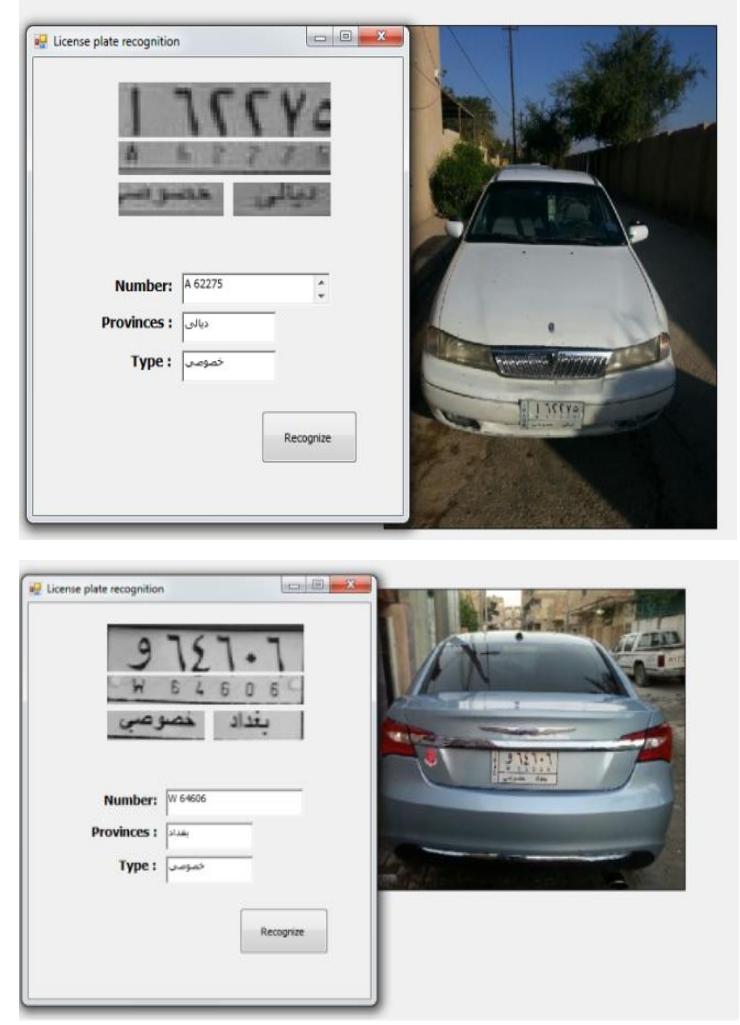

Fig. 10. Segmented characters.

\section{CONCLUSIONS}

We designed in this paper a recognition application of new Iraq's vehicle plates has been designed. The image of plate processed and gets plate locations. Then, we separated it by segmentation.
Finally, we applied algorithm of template matching for recognition of plate image segments with all possibilities such as Arabic, Indian numbers, English and Arabic letters and in addition to Arabic words in template data base. This designed application was examined over a 30 images. Through recognition experiments, it was proven that the designed system is better than $88 \%$ recognition rates. This because of the effects of image noise, such as the adhesion and fracture on the plates, it is necessary to make further selection of multi features to characters. and the location of plates backgrounds also need further study so the new license plate is very difficult to extract and recognition because the Iraq plate have 6 parts as we show in this paper. Finally, the segmentation of the plate is also difficult to separate these six parts because plate characters' extraction needs high accuracy when working on images of busy roads or parking areas. This system gives about $88 \%$ of efficiency is a good result.

\section{REFERENCES}

[1] Christos NE. Anagnostopoulos, IE. Anagnostopoulos, VL, Eleftherios K. A license platerecognition algorithm for intelligent transportation system applications. Browse Journals \& Magazines 2006; 7 (3): 377-392.

[2] Öztürk F, Özen F. A new license plate recognition system based on probabilistic neural networks. Procedia Technology 2012; 1: 124-128.

[3] Roy A, Ghoshal DP. Number plate recognition for use in different countries using an improved segmenation. IEEE in 2nd National Conference on Emerging Trends and Applications in Computer Science (NCETACS) 2011, 4-5 March; India: pp. 15.

[4] Chen Z-X, Liu C-Y, Chang F-L, Wang G-Y. Automatic license-plate location and recognition based on feature salience. IEEE Transactions on Vehicular Technology 2009; l (7): 3781-3785.

[5] Wen $\mathrm{Y}$ et al. An algorithm for license plate recognition applied to intelligent transportation system. IEEE Transactions of Intelligent Transportation Systems 2011; 12 (3): 1-16.

[6] Jia W, Zhang H, He X. Region-based license plate detection. Journal of Network and Computer Applications 2007; 30 (4): 1324-1333.

[7] Yang Y, Gao X, Yang G. Study the method of vehicle license locating based on color segmentation. Procedia Engineering 2011; 15: 1324-1329.

[8] Wang F et al. Fuzzy-based algorithm for color recognition of license plates. Pattern Recognition Letters 2008; 29 (7): 1007-1020.

[9] Kamal NN. Iraqi license plate recognition system: Baghdad University, Baghdad, Iraq: 2013.

[10] Jones, PV. Robust real-time object detection. International Journal of Computer Vision 2004; 57 (2): 137-154.

[11] Paul V, Michael J. Rapid object detection using a boosted cascade of simple features. IEEE Conference on Computer Vision and Pattern Recognition 2001, December. 